\title{
Simplification of earthquake accelerograms for quick nonlinear time history analyses by using modified Inverse Fourier Transform
}

\author{
A. Faroughi ${ }^{1}$ \& M. Hosseini ${ }^{2}$ \\ ${ }^{I}$ Department of Civil Engineering, East Tehran Branch, \\ Islamic Azad University, Tehran, Iran \\ ${ }^{2}$ Structural Engineering Research Center, \\ International Institute of Earthquake Engineering and Seismology \\ (IIEES), Tehran, Iran
}

\begin{abstract}
In seismic design and evaluation of irregular buildings most codes recommend nonlinear time history analysis (NLTHA), which is very time-consuming, mainly since the time step size of the digitized accelerograms is usually very small. Therefore, if the NLTHA analysis can be performed by large time steps without losing much precision, it can be very helpful. This paper introduces a method for simplification of accelerograms based on the modification of their Fourier analyses. In the proposed method the Fourier Spectrum of the accelerogram is calculated first, and then, by using a computer program, developed by the authors, the corresponding Inverse Fourier Transform is calculated using a relatively large time step, depending on the structure's periods, which is usually 5 to 10 times of the original accelerogram's time step) to create the simplified accelerogram. Obviously, time history analysis by using this simplified accelerogram is much less time-consuming. Numerical results show that the maximum response values obtained by using the simplified accelerograms have only 5 to 10 percent error, depending on the type of the structure and the features of the used records.
\end{abstract}

Keywords: Fourier and Inverse Fourier Transforms, time step size. 


\section{Introduction}

In seismic design and evaluation of many kinds of structures, including irregular building systems, there are several cases in which the simplified seismic analysis procedures suggested by seismic design codes, are not usable. Irregular buildings, buildings with more than 15 stories (according to most of codes), and many special structures are some of these cases. In such cases most codes recommend nonlinear time history analysis (NLTHA) as the most appropriate analysis procedure. However, NLTHA is usually very time-consuming and therefore costly, basically, because of the very small size of the time step used in digitization of accelerograms. On this basis, if the time history analysis can be performed by relatively larger time steps, without losing much precision, it will be very helpful. Using simplified accelerograms, as discussed by Wang [1] in 1975 and Wang and Goel [2] in 1977 is an approach for this purpose. In these two works the real accelerogram has been condensed into a four-pulse model by a minimization method, using rectangular pulses. Creating the possibility of using larger time steps, by some techniques as proposed by Soroushian [3] in 2008 , is another approach proposed for this purpose. Simplification of digitized accelerograms, however, can be done by some other means such as Fourier and Inverse Fourier Transforms, so that the simplified accelerogram can have much larger time steps.

In this paper a method is introduced for simplification of accelerograms based on the modification of their Fourier analyses. For this purpose, at first the Fourier Spectrum of the accelerogram is calculated, by a computer program, developed by the authors. Then, by using the developed computer program the corresponding Inverse Fourier Transform is calculated, using a relatively large time step, depending on the period of the highest used frequency (which is usually 5 to 10 times larger than the original accelerogram's time step) to create the simplified accelerogram. The use of accelerograms, simplified by the proposed method, in case of linear systems, and the good precision of the calculated responses have been presented in a previous paper of the authors, Faroughi and Hosseini, [4], in 2010. The details of this technique are described in the following section of the paper, and its efficiency in reducing the required time for the cases of nonlinear time history analysis (NLTHA) is also shown by some numerical examples.

\section{Accelerogram simplification and sample results}

As mentioned in the previous section, the simplification technique is based on modifying the Fourier Transform of the original digitized accelerogram and then calculating its Inverse Fourier Transform using time step size much larger than that of the original digitized accelerogram. For performing this modification, a computer program has been developed by the authors, which is compatible with Microsoft Office program. In this computer program at first the Fourier Transform of the ground acceleration time history, $a_{g}(t)$, is calculated by: 


$$
F(\omega)=\int_{0}^{\tau_{0}} a_{g}(t) e^{-i \omega t} d t
$$

where $\tau_{0}$ is the duration of the accelerogram. Regarding that $F(\omega)$ has a real part and an imaginary part as:

$$
\begin{aligned}
& C(\omega)=\int_{0}^{T_{0}} a_{g}(t) \cos \omega t d t \\
& S(\omega)=\int_{0}^{T_{0}} a_{g}(t) \sin \omega t d t
\end{aligned}
$$

Then, the Fourier amplitude or spectral value can be calculated as:

$$
F A S(\omega)=\sqrt{C^{2}(\omega)+S^{2}(\omega)}
$$

A sample page of the screen view of the developed program for Fourier and Inverse Fourier Transforms for simplification of accelerograms is shown in Figure 1, in which the accelerogram of Tabas, Iran, earthquake of 1978, which has been digitized with a time step size of $0.01 \mathrm{sec}$, and its Fourier Transform are shown.

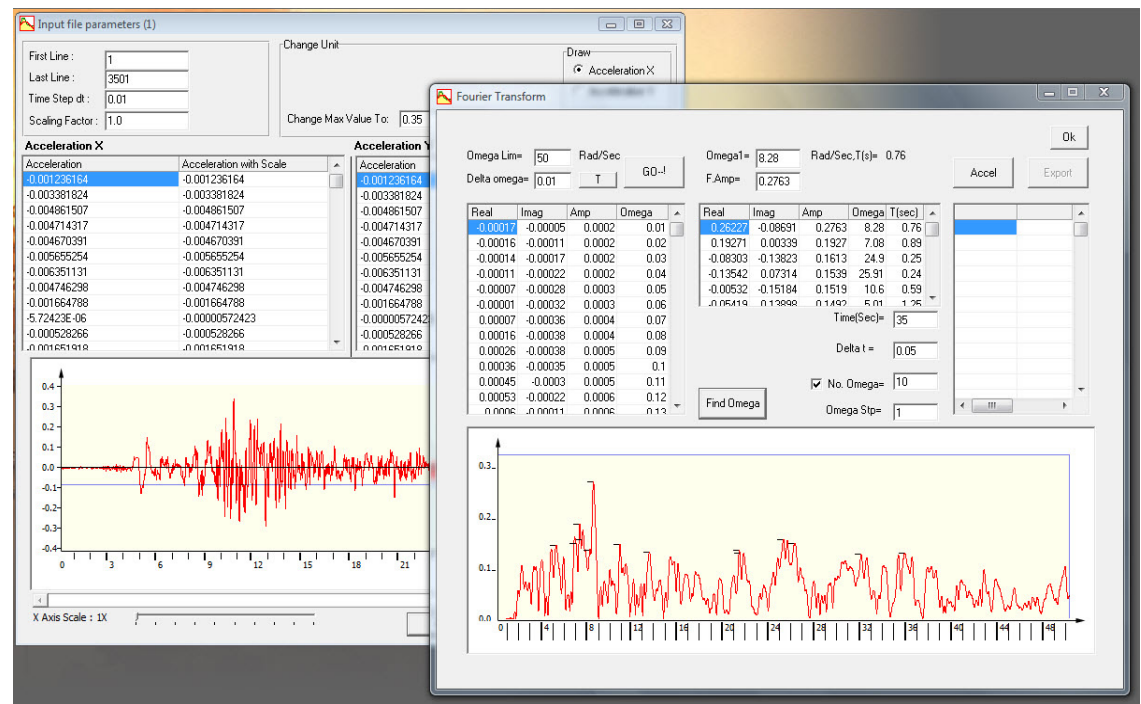

Figure 1: A screen view of the developed computer program for simplification of digitized accelerograms. 
For obtaining the simplified accelerogram a larger time step such as $0.05 \mathrm{sec}$ is considered and the modified Inverse Fourier Transform of the record is calculated. The results of these calculations, relating to one of the accelerograms of Chi-Chi, Taiwan, earthquake of 1999 , with the time step of $0.005 \mathrm{sec}$, and its simplified version by using a time step size of $0.05 \mathrm{sec}$, which is ten times of the time step size of the original record is shown in Figure 2.

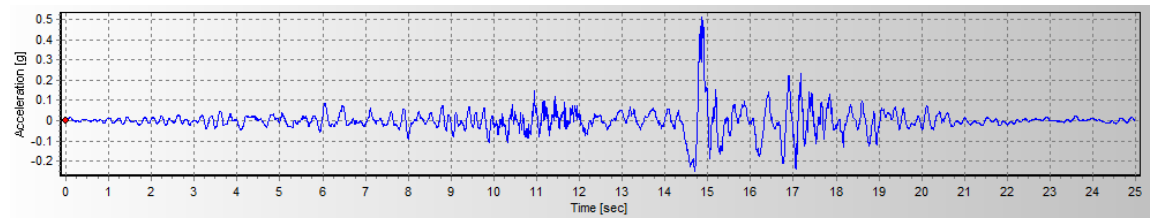

(a)

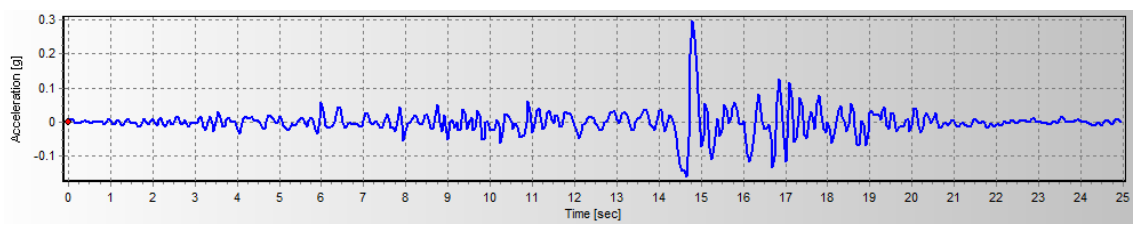

(b)

Figure 2: $\quad$ The original (a) and the simplified (b) accelerograms of one of ChiChi, Taiwan earthquake records.

In Figure 2, it can be seen that the two records have very similar pattern, however, the simplified record has a little lower peak values. The reason behind this reduction is the use of larger time step size, which results in the omission of some high frequency oscillations of the record.

\section{Sample numerical results of nonlinear responses}

To see whether the simplified accelerograms result in response values with acceptable precision, the displacement response and also the hysteretic base shear - lateral displacement curves of a set of single degree of freedom (SDOF) systems, having natural periods of $0.3,0.5,0.7$, and $0.9 \mathrm{sec}$ were calculated by both original and simplified records of a few earthquakes, including Chi-Chi and Loma Prieta. As samples of results the responses related to the system with the natural period of $0.5 \mathrm{sec}$, subjected to Chi-Chi and Loma Prieta earthquakes are shown in Figures 3 to 6. 


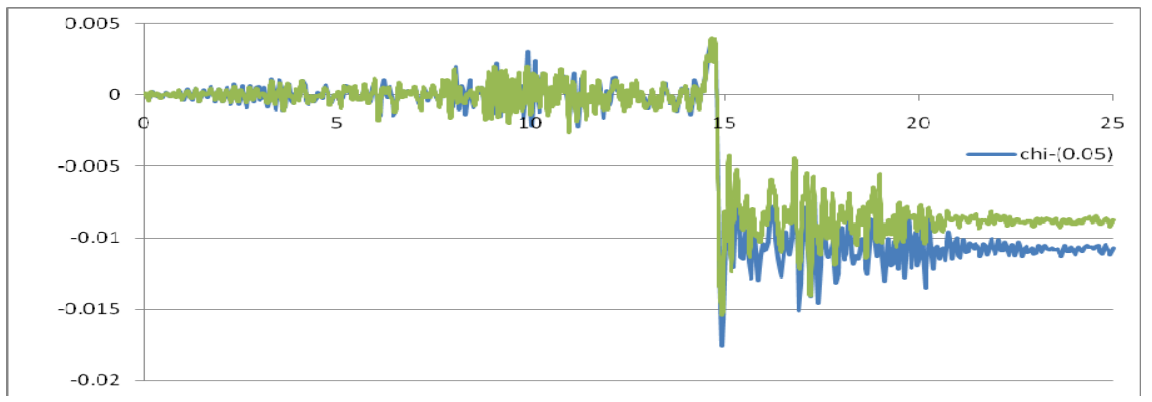

Figure 3: Displacement response histories of the nonlinear SDOF system, having the natural period of $0.5 \mathrm{sec}$, calculated by both original and simplified record (shown in blue) of Chi-Chi earthquake.

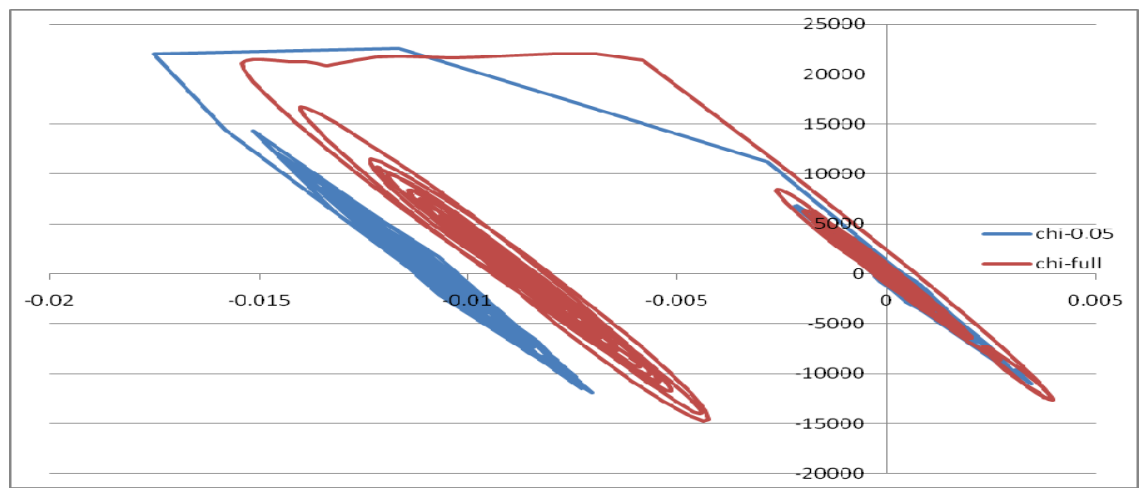

Figure 4: Hysteretic responses of the nonlinear SDOF system, having the natural period of $0.5 \mathrm{sec}$, calculated by both original and simplified record (shown in blue) of Chi-Chi earthquake.

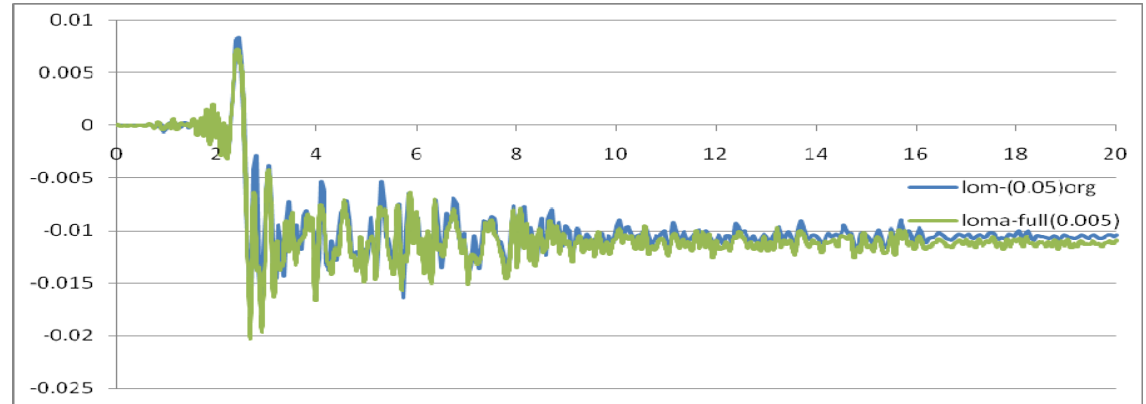

Figure 5: Displacement response histories of the nonlinear SDOF system, having the natural period of $0.5 \mathrm{sec}$, calculated by both original and simplified record (shown in blue) of Loa Prieta earthquake. 


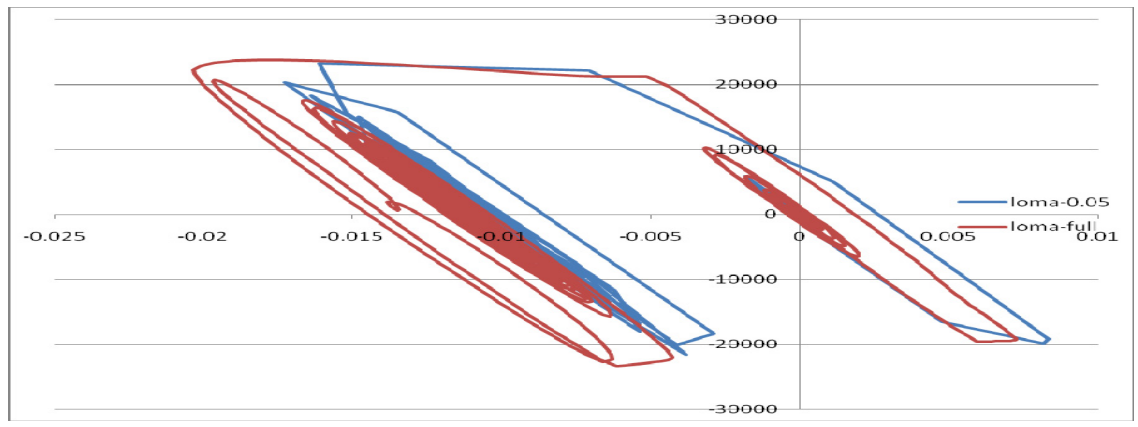

Figure 6: Hysteretic responses of the nonlinear SDOF system, having the natural period of $0.5 \mathrm{sec}$, calculated by both original and simplified record (shown in blue) of Loma Prieta earthquake.

The good agreement of response values, obtained by the original and the simplified accelerogram can be observed in Figures 3 to 6 . It can be seen also in this figures that the displacement responses obtained by the simplified accelerograms are a little higher than those obtained by the original records in cases of both Chi-Chi and Loma Prieta earthquakes. This is true in case SDOF system with other values of natural period subjected to other earthquakes as well, and this shows that the proposed simplification technique results in conservative responses.

To make sure that the agreement exists in the whole frequency range of the earthquakes for acceleration responses as well, the Fourier amplitude, the pseudo velocity and also the pseudo acceleration spectra of both original and simplified records were calculated. Samples of these spectra are shown in Figures 7 to 9, which are related to accelerogram of Chi-Chi earthquake and its simplified record using a time step size of $0.03 \mathrm{sec}$, which is 6 times of the time step size of the original record.

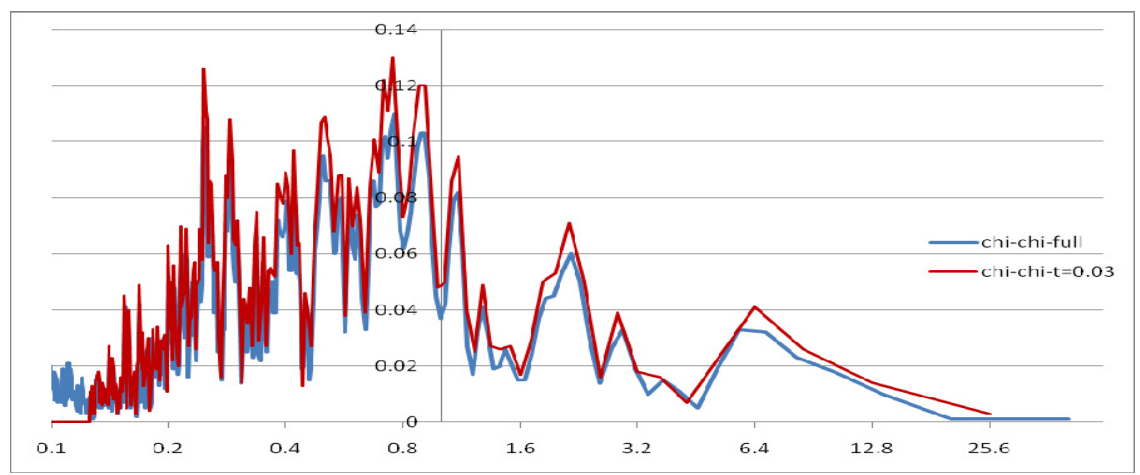

Figure 7: Fourier amplitude spectra of the considered accelerogram of ChiChi earthquake calculated by both original and simplified records, using a time step size of $0.03 \mathrm{sec}$ ( 6 times of the original record). 


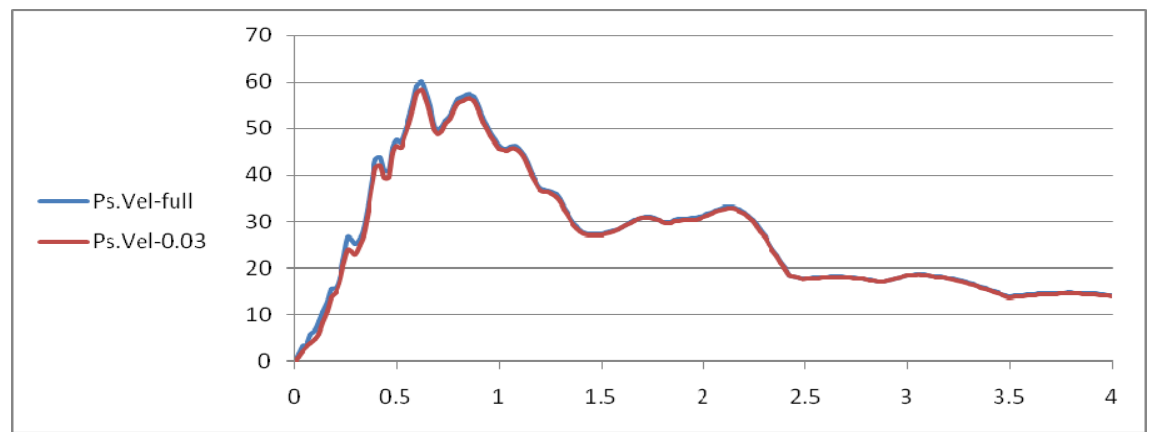

Figure 8: $\quad$ Pseudo velocity spectra of the considered accelerogram of Chi-Chi earthquake calculated by both original and simplified records, using a time step size of $0.03 \mathrm{sec}$ (6 times of the original record).

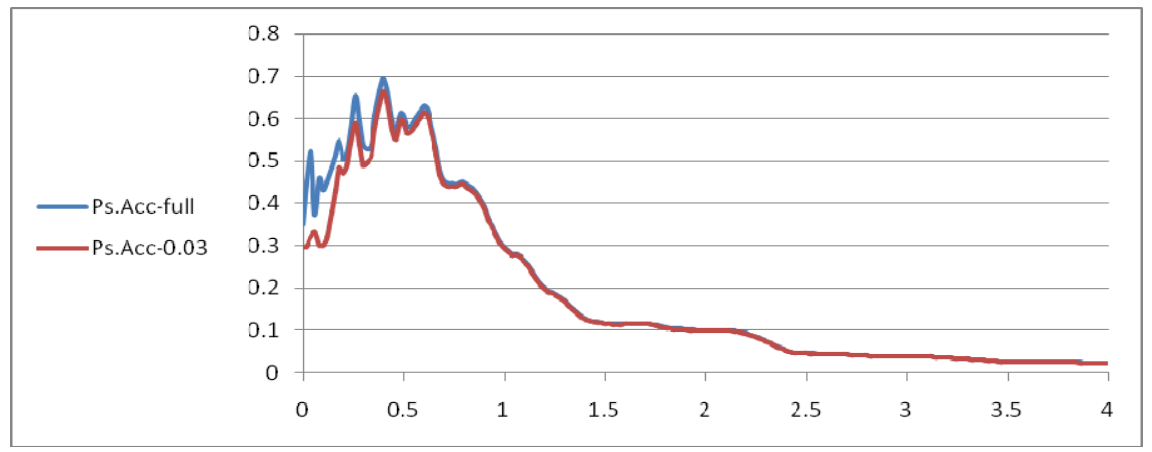

Figure 9: Pseudo acceleration spectra of the considered accelerogram of ChiChi earthquake calculated by both original and simplified records, using a time step size of $0.03 \mathrm{sec}$ ( 6 times of the original record).

It can be seen in Figures 7 to 9 that a very good agreement exists between the Fourier amplitude, pseudo velocity and pseudo acceleration spectra in almost the whole frequency range. As expected, in case of pseudo acceleration spectra the agreement is a little weak in the range of very lower periods, or higher frequencies.

\section{Conclusions}

Numerical results show that simplification of records by reproducing their Inverse Fourier Transform using a time step size of 5 to 10 times of that of the original records leads to only $5-10 \%$ overestimation of the maximum values of displacement and base shear force, depending on the natural period of the SDOF system and the features of the used records, while decreases the required response calculation time up to 10 times. With regard to response spectra, the 
simplified records leads to some reduction of the spectral acceleration values in lower period values, however the pseudo velocities of the original and the simplified records are in good agreement in the whole period range. Based on these results, it can be claimed that the proposed simplification technique is quite effective in reducing the computation costs of nonlinear time history analyses.

\section{References}

[1] Wang, W.Y.L., Structural instability during earthquakes and accelerogram simplification, Ph.D. Thesis, Michigan University, Ann Arbor, USA, 1975.

[2] Wang, Warren Y.L. and Goel, Subhash C., Prediction of maximum structural response by using simplified accelerograms, Proceedings of the $6^{\text {th }}$ World Conference on Earthquake Engineering, New Delhi, India, 1977.

[3] Soroushian, A., A technique for time integration analysis with steps larger than the excitation steps, Communications in Numerical Methods in Engineering, Special Issue: Numerical Modeling of Carbon-based Material Systems and Related Topics, 24(12), pp. 2087-2111, 2008.

[4] Faroughi, Alireza and Hosseini, Mahmood, Simplification of Earthquake Accelerograms for Quick Time History Analyses by Using Their Modified Inverse Fourier Transforms, Proceedings of the Twelfth East Asia-Pacific Conference on Structural Engineering and Construction (EASEC-12), Hong Kong Special Administrative Region, China, 24-26 January 2011. 